\section{Modafinil modulates anterior cingulate function in chronic schizophrenia}

\author{
SEAN A. SPENCE, RUSSELL D. GREEN, IAIN D. WILKINSON \\ and MIKE D. HUNTER
}

Schizophrenia is associated with widespread cognitive impairments, some of which, such as those affecting memory and vigilance, have an impact upon independent living (Velligan et al, 1997). Amelioration of such impairments might improve the quality of life, therefore we studied the effects of a putative cognitive enhancer, modafinil, upon prefrontal function in people with chronic schizophrenia. Modafinil (2-[(diphenylmethyl)sulphinyl] acetamide) is a novel agent that promotes wakefulness and is licensed in the UK and USA for the treatment of narcolepsy (Cephalon, 1999; US Modafinil in Narcolepsy Multicenter Study Group, 2000; British Medical Association, 2004). Its precise mode of action is uncertain, although in animal models it has been shown to elicit 'early gene' expression in anterior hypothalamus (Lin et al, 1996) and anterior cingulate cortex (Scammell et al, 2000), findings congruent with its properties. Very recently, modafinil has been reported to enhance short-term verbal memory in people with schizophrenia (Turner et al, 2004).

\section{METHOD}

On the basis of animal studies, we chose to probe cognitive functions that would engage 'higher' executive regions, particularly the anterior cingulate cortex (implicated in vigilance). However, in view of the uncertain mechanism of action of modafinil, and emerging evidence of a possible risk of psychosis exacerbation (Narendran et al, 2002), we utilised a relatively small dose of drug. Our study was conducted between 28 August 2002 and 4 July 2003 .

\section{Participants}

Right-handed males aged 18-60 years, with premorbid IQ $>70$ on the National Adult Reading Test (NART; Nelson \& O'Connell, 1978) and a DSM-IV
(American Psychiatric Association, 1994) diagnosis of schizophrenia and prominent negative symptomatology (rating 3 on at least one item of the Scale for the Assessment of Negative Symptoms (SANS); Andreasen, 1983) were included. Exclusion criteria were: prominent 'positive' symptomatology (marked delusions and/or hallucinations); recent history of mental state instability; changes to psychotropic medication or admission to hospital within 3 months of assessment; significant history of neurological, endocrine or cardiovascular disorder; hypersensitivity to modafinil; concurrent prescription of other stimulant medication; concurrent substance misuse; and contraindications to magnetic resonance imaging scanning (metallic implants, foreign bodies and claustrophobia).

Thirty-two patients were approached and 21 agreed to participate following a full explanation of the study. Nineteen of these satisfied detailed assessment of the inclusion and exclusion criteria. Participants underwent psychiatric (Brief Psychiatric Rating Scale; Overall \& Gorham, 1962; SANS; Scale for the Assessment of Positive Symptoms; Andreasen, 1984; Beck Depression Inventory; Beck et al, 1961; MiniMental State Examination; Folstein et al, 1975), physical (including an electrocardiogram) and neuropsychological assessment (Simpson-Angus Scale; Simpson \& Angus, 1970; Abnormal Involuntary Movements Scale; Guy, 1976; Barnes Akathisia Scale; Barnes, 1989).

Written informed consent was obtained from each patient. The study was approved by the North and South Sheffield Research Ethics Committees, and also the Rotherham and the Doncaster and South Humber Research Ethics Committees. Because the study was funded by an investigator-led award' and was not a 'company-sponsored trial', research indemnity was provided by the Sheffield Care Trust (and reciprocally participating National Health Service trusts) and clinical trial insurance was provided by the University of Sheffield.

\section{Procedures}

We utilised a randomised, double-blind placebo-controlled crossover design. Patients were studied on 2 days, 1 week apart. On each day, patients received oral modafinil $(100 \mathrm{mg})$ or placebo $2 \mathrm{~h}$ prior to functional magnetic resonance imaging (fMRI) scanning. Administration and scanning times were predicated on the drug's pharmacokinetics 
in humans; peak plasma levels occur 2-4h post-acute oral dosing (Cephalon, 1999; Robertson \& Hellriegel, 2003). Randomisation, performed by a pharmacist (not a member of the research team), was achieved by drawing labelled counters; this ensured that approximately equivalent numbers of patients received modafinil and placebo on day 1 , and vice versa on day 2. Patients were required not to smoke or consume caffeine prior to scanning. They were admitted for 24-h observation after the scanning procedure. The outcome measures were: a difference in fMRI signal, during a working memory task, between the modafinil and placebo conditions; a difference in behavioural performance (accuracy), during the same intra-scanner task, between modafinil and placebo conditions; and a patient-wise bivariate correlation between the first two measures.

\section{Psychological paradigm}

Inside the scanner, patients performed a standard working memory task (the '2 back'; Callicott et al, 1998). This difficult task required subjects to monitor, update and temporally 'tag' the contents of their working memory (Manoach, 2003). A series of numbers (between 1 and 4) was presented visually, in a pseudo-random order, one every $2 \mathrm{~s}$. Colour coding of these stimuli cued patients to indicate (by pressing a button) either which number was currently presented on the screen (the ' 0 -back'; baseline condition) or which number had been presented two trials earlier (i.e. the '2-back'; active condition). Stimuli were delivered using Presentation (Neurobehavioral Systems Inc, California, USA) software running on a personal computer via a video projector and mirror located inside the scanner bore. Patients responded by pressing one of four buttons on an intrascanner box optically connected to the computer system via an interface (New Micros Inc, Texas, USA). In an alternating, blocked 0-back/2-back design, 15 consecutive '0-back' stimuli (lasting 30 s) were followed by 15 consecutive '2-back' stimuli (also lasting $30 \mathrm{~s}$ ). This sequence was repeated six times; hence the functional scans lasted $6 \mathrm{~min}$ in total.

Patients practised the task prior to entering the scanner but did so only three times in order to minimise any automation of the procedure. Different performance levels have been permitted in previous studies. Some have incorporated a wide range of accuracy among patients with schizophrenia (Callicott et al, 1998, where performance was relatively poor; MeyerLindenberg et al, 2001; Bertolino et al, 2003) whereas others have set more stringent thresholds (Callicott et al, 2000, 2003). Comparison with a healthy control group necessarily requires comparable levels of performance across the groups, but in our study we wished to compare patients' performances against themselves (on and off modafinil). Hence, we allowed for a range of performance accuracy. This was partly pragmatic, given that we were deliberately studying people with chronic schizophrenia and prominent negative symptomatology (the likely recipients of putative cognitive enhancers), but also design-led because we required a range of performance across the group to detect changes in performance within subjects exposed to modafinil (hence avoiding 'ceiling effects') and to allow for post hoc correlations with performance per se (Manoach et al, 1999; Callicott et al, 2000, 2003).

\section{Functional image acquisition and analysis}

At each of 120 functional imaging time points, $32 \times 4 \mathrm{~mm}$ contiguous $\mathrm{T}_{2}{ }^{*}$-weighted slices were acquired using echo-planar imaging on a $1.5 \mathrm{~T}$ system (Eclipse, Philips, Ohio) at Sheffield University (repetition time $=3 \mathrm{~s}$; echo time $=40 \mathrm{~ms}$; field of view $=$ $240 \mathrm{~mm}$; in-plane matrix $=128 \times 128$ ).

Images were analysed using SPM99 (http://www.fil.ion.ucl.ac.uk/). The blood oxygenation level-dependent (BOLD) response that is measured by fMRI is thought to represent a vascular marker of neuronal activation (Logothetis et al, 2001). Following timing and movement correction, spatial normalisation and smoothing with a Gaussian kernel of $6 \mathrm{~mm}$ full width at half-maximum (Friston et al, 1995), we used a 'boxcar' wave convolved with a synthetic haemodynamic response function to model the BOLD response. Each patient had two fMRI data-sets (modafinil and placebo); for each individual data-set, a first-level voxel-wise contrast of activation during the working memory $v$. baseline was undertaken. This generated contrast images, which were then used in a secondorder (random-effects) group analysis.

Random-effects analyses allow quantitative inferences to be drawn regarding the average behaviour of the population from which patients are selected, across different scanning sessions. Such an analysis is mandatory in psychopharmacological designs where the main effect of interest exists only between distinct scanning sessions (i.e., modafinil $v$. placebo; Friston et al, 1999). Individual contrast images were entered as data points in a wholegroup analysis of covariance (ANCOVA) that compared brain activation during working memory $v$. baseline conditions on modafinil with activation during working memory $v$. baseline on placebo. Order of scanning (whether modafinil was received on day 1 or day 2) comprised the 'nuisance' covariate. This produced a group parametric brain map of $t$-statistics, in the stereotactic space of the Montreal Neurological Institute (MNI; Evans et al, 1993), showing brain areas more activated during working memory than baseline conditions on modafinil compared with placebo. We emphasise that intra-session effects (working memory $v$. baseline) were modelled at the first level but that the main inter-session effect (modafinil $v$. placebo) was modelled in the second-level (random effects) analysis.

We also produced two subsidiary brain maps, demonstrating brain areas more activated during working memory than baseline on placebo only and modafinil only, using two single group ANCOVAs (as before, with scanning order as 'nuisance' covariate). Hence, we were able to ensure that the expected pattern of cortical activation was obtained during the '2-back' irrespective of drug/placebo condition.

Because our study was hypothesis driven, we set our significance threshold at $P<0.01$, uncorrected for height and extent of activation. We also designated a small volume (sphere of diameter= $10 \mathrm{~mm}$ ) that could be used to correct for multiple comparisons (family-wise error method) should activation in the a priori region of interest (anterior cingulate cortex) be observed at the uncorrected threshold. For the purposes of reporting and neuroanatomical labelling, the stereotactic coordinates of activated areas were transformed from MNI space into the system of Talairach \& Tournoux (1988).

\section{Brain activation/behavioural performance correlations}

For each patient we calculated a measure of performance (percentage accuracy) during the working memory task on both study days, and hence a 'difference' score 
between the modafinil and placebo conditions. Taking the anterior cingulate cortex as a region of interest, we also estimated the magnitude of fMRI signal change (derived from the 'beta' parameters in SPM99) during the working memory task ( $v$. baseline) on both study days. For each patient, this allowed us to calculate the difference in the anterior cingulate cortex signal change, during the working memory task, between modafinil and placebo conditions. We then ran patientwise bivariate correlations between the difference in behavioural performance on modafinil $v$. placebo and the difference in anterior cingulate cortex signal change on modafinil $v$. placebo.

\section{RESULTS}

\section{Adverse events and image quality}

We analysed data from 17 of the 19 patients entering the study. One patient did not complete the study due to relapse of acute psychosis 4 days after the first scanning day. Ethical considerations required disclosure of his randomisation status, revealing that he had received modafinil. This was the only clinically significant adverse event that occurred during the study. Data from another patient could not be analysed because of technical problems with his second-session fMRI images.

\section{Demographic data}

Patients were predominantly middle-aged males who had been ill for approximately 15 years (Table 1). Sixteen were single; none was in paid employment but five performed voluntary work; 16 received maximum disability living allowance; eight lived in their 'own' accommodation; three with parents, two in supported group projects and four in rehabilitation units. Most patients were receiving oral antipsychotics $(n=13)$, which were 'atypical' in all but one case. Four patients received intramuscular ('typical') depot medication. Of these 17 patients, none changed medication during the study period. Nine patients received modafinil on day 1 and placebo on day 2; eight received the reverse.

\section{Behavioural measures}

Statistical comparisons were prespecified, except where indicated. We used nonparametric tests to analyse the group behavioural (accuracy) data during the
Table I Demographic and clinical characteristics of included patients

\begin{tabular}{lcc}
\hline & Mean (maximum possible) & s.d. \\
\hline Age (years) & 37.7 & 9.5 \\
Years of education & 10.9 & 2.7 \\
IQ & 104.8 & 9.4 \\
Edinburgh Handedness Inventory & $100 \%$ right-handed & 0 \\
& & \\
Clinical features & & 14.8 \\
Duration of illness (years) & $55.2(112)$ & 10.1 \\
Brief Psychiatric Rating Scale & $11.5(25)$ & 10.5 \\
Scale for the Assessment of Negative Symptoms & $3.6(20)$ & 2.2 \\
Scale for the Assessment of Positive Symptoms & $10.3(63)$ & 2.1 \\
Beck Depression Inventory & $29.3(30)$ & 10.7 \\
Mini-Mental State Examination & & 0.6 \\
& & 2.6 \\
Extrapyramidal side-effects & & \\
Simpson-Angus Scale & $6.1(40)$ & $2.3(40)$ \\
Abnormal Involuntary Movements Scale & $1.9(14)$ & 3.2 \\
Barnes Akathisia Scale & & 2 \\
\hline
\end{tabular}

working memory task under placebo and modafinil conditions (these were not normally distributed). Under placebo conditions, response accuracy for the control ' 0 -back' condition was $19-88 \%$ (median $71 \%$ ) and did not differ significantly from that on modafinil: range 11-99\%, median $71 \%$ (Wilcoxon signed rank test; $Z=0.12$; $P=0.91$ ). Under placebo conditions, response accuracy for the '2-back' task was $5-85 \%$ (median 26\%) and did not differ significantly from that on modafinil: range 4-79\%, median 22\% (Wilcoxon signed rank test; $Z=0.97 ; P=0.33$ ).

\section{Brain activations}

Functional image analysis showed that on both the modafinil and placebo days the patients exhibited activations during the 2-back condition (relative to the 0 -back) in predicted brain regions (Tables 2 and 3), specifically the prefrontal, anterior cingulate and parietal cortices (Callicott et al, 2000, 2003; Meyer-Lindenberg et al, 2001).

Functional image analysis comparing the modafinil with the placebo condition revealed that working memory task performance was associated with significantly
Table 2 Brain areas exhibiting greater activation during working memory than baseline under placebo condition

\begin{tabular}{lccc}
\hline Region (Brodmann area) & Coordinates $(x, y, z)$ & $Z$ & Voxels \\
\hline Left middle frontal gyrus (9/46) & $-50,32,21$ & 3.56 & 159 \\
Left middle/superior frontal gyrus (6/8) & $-26,24,54$ & 3.42 & 151 \\
Anterior cingulate gyrus (32) & $0,22,45$ & 3.90 & 173 \\
Right inferior frontal gyrus (47) & $50,17,-9$ & 3.99 & 132 \\
Right middle frontal gyrus (6) & $36,3,57$ & 3.67 & 149 \\
Left inferior parietal lobule (40) & $-48,-45,41$ & 3.63 & 135 \\
Right inferior parietal lobule (40) & $42,-50,54$ & 4.10 & 194 \\
Right posterior parietal cortex (7/19/40) & $34,-66,36$ & 4.23 & 172 \\
Right cerebellar hemisphere & $38,-71,-28$ & 3.27 & 101 \\
\hline
\end{tabular}

Activations exceeding statistical threshold $P<0.01$, uncorrected, for height and extent of activation are shown; stereotactic coordinates are in the standard space of Talairach \& Tournoux (1988); ' $Z$ ' reflects peak statistical 'height' of the most activated voxel in each cluster; 'voxels' indicates total number of voxels exceeding height threshold $P<0.0$ I in each cluster. 
Table 3 Brain areas exhibiting greater activation during working memory than baseline under modafinil condition

\begin{tabular}{lccc}
\hline Region (Brodmann area) & Coordinates $(x, y, z)$ & $Z$ & Voxels \\
\hline Left inferior/middle frontal gyrus (44/10) & $-38,52, I$ & 4.36 & 502 \\
Right anterior cingulate gyrus (32) & $6,38,18$ & 3.79 & $50^{\prime}$ \\
Right inferior frontal gyrus (47) & $38,23,-11$ & 4.14 & 305 \\
Right middle/superior frontal gyrus (6/8) & $34,10,51$ & 5.27 & $6165^{2}$ \\
Right anterior cingulate/medial frontal gyrus (32/8) & $4,27,35$ & 5.25 & 2 \\
Left inferior frontal gyrus (47) & $-30,21,-3$ & 4.85 & 2 \\
Left posterior parietal cortex (19) & $-28,-68,42$ & 5.03 & $4439^{2}$ \\
Left precuneus (7) & $-6,-65,51$ & 4.56 & 2 \\
Right posterior parietal cortex (19) & $32,-64,33$ & 4.49 & 2 \\
Right cerebellar vermis & $10,-83,-21$ & 4.08 & 145 \\
Right cerebellar hemisphere & $36,-69,-25$ & 3.89 & 305 \\
Left cerebellar hemisphere & $-30,-62,-30$ & 3.82 & 176 \\
\hline Activtions
\end{tabular}

Activations exceeding statistical threshold $P<0.01$, uncorrected, for height and extent of activation are shown; stereotactic coordinates are in the standard space of Talairach \& Tournoux (1988); ' $Z$ ' reflects peak statistical 'height' of the most activated voxel in each cluster; 'voxels' indicates total number of voxels exceeding height threshold $P<0.01$ in each cluster.

I. Although not significant for extent of activation in the modafinil-only analysis, this distinct area of anterior cingulate cortex activation represents the region of 'most difference' between modafinil and placebo conditions and, hence, corresponds to the basis for the activation observed in the main modafinil $v$. placebo contrast (the coordinates were also used to define a region of interest within which correction for multiple comparisons was performed; see text and Fig. I). 2. Large clusters and their anatomically distinct foci.

greater activation solely in the anterior cingulate cortex (Fig. 1; Talairach coordinates: $x=6 \mathrm{~mm}, y=38 \mathrm{~mm}, z=15 \mathrm{~mm} ; 131$ supra-threshold voxels; peak $Z$ score $=3.14$; $P<0.01$, uncorrected, for height and extent of activation). This activation remained significant after correction for multiple comparisons within the defined region of interest $(P<0.05)$. Examining the separate modafinil and placebo brain maps (of the working memory $v$. baseline contrast) confirmed that this focal difference was due to greater anterior cingulate cortex activation during working memory than baseline performance on modafinil $(x=6 \mathrm{~mm}, y=38 \mathrm{~mm}, z=18 \mathrm{~mm} ; 50$ voxels; $Z=3.79 ; P<0.01$, uncorrected, for height of activation), and not the converse, namely, greater activation during baseline than working memory on placebo.

Patient-wise differences in anterior cingulate cortex activation (Fig. 1) and behavioural performance (between modafinil and placebo conditions) were positively and significantly correlated (Fig. 2; Spearman's $\rho=0.42$; one-tailed $P<0.05$ ). However, this correlation did not reflect a straightforward relationship between improved activation and improved performance. Rather, it reflected an increased fMRI signal in the majority of patients during the modafinil session, with concomitant improvement of memory performance in half and a decreased signal in a inil), most of whom exhibited reduced performance on the drug (Fig. 2). Hence, (a) Coronal view

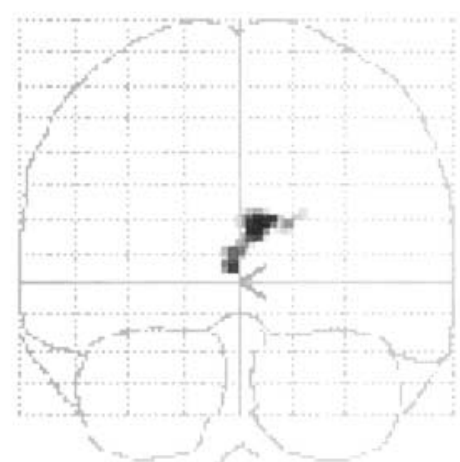

for the patient group as a whole there was a relationship between the degree to which anterior cingulate cortex activation, during the memory task, was modulated (increased/ decreased) by the drug and their level of cognitive performance. Although a subgroup of patients exhibited enhanced cognition (in association with increased anterior cingulate cortex activation), no patient exhibiting reduced anterior cingulate cortex activity improved cognitively.

Post hoc examination of the data revealed that of the five patients exhibiting improvements of both anterior cingulate cortex fMRI signal and 2-back performance, four were receiving 'typical' neuroleptics (depot or sulpiride) and one was receiving olanzapine. That is, of five patients who received 'typicals' the majority responded positively to modafinil (both physiologically and cognitively). Conversely, of 12 patients receiving 'atypicals' only one exhibited this pattern. Moreover, a post hoc analysis confirmed that the positive correlation between anterior cingulate cortex signal and performance change was most significant in the five patients receiving 'typical' antipsychotics (Spearman's $\rho=1.00, P$ not calculated). Conversely,

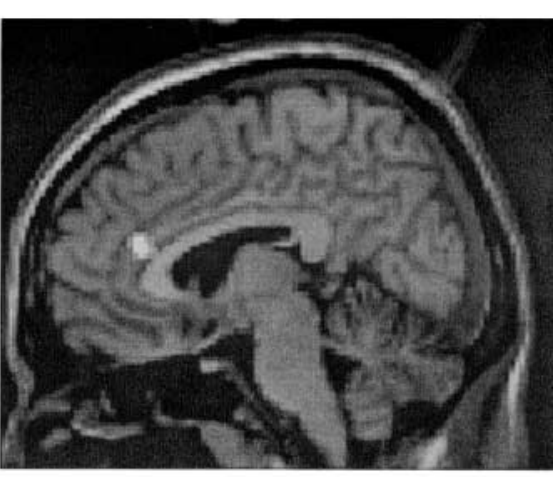

(b) Sagittal view

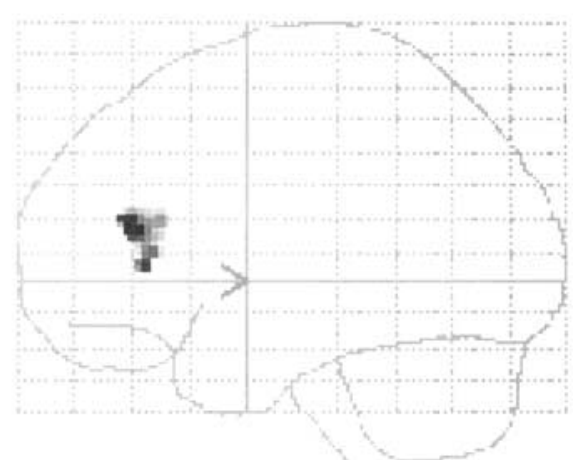

Fig. I The brain area (anterior cingulate cortex) that exhibited greater activation during a working memory task on modafinil than on placebo. Group data displayed against a 'canonical' $T_{1}$-weighted image (upper panels); supra-threshold voxels are shown in yellow $(P<0.0 \mathrm{l})$. In order to demonstrate the regional specificity of this finding the data are also displayed at the same statistical threshold within a 'glass brain' (lower panels). 


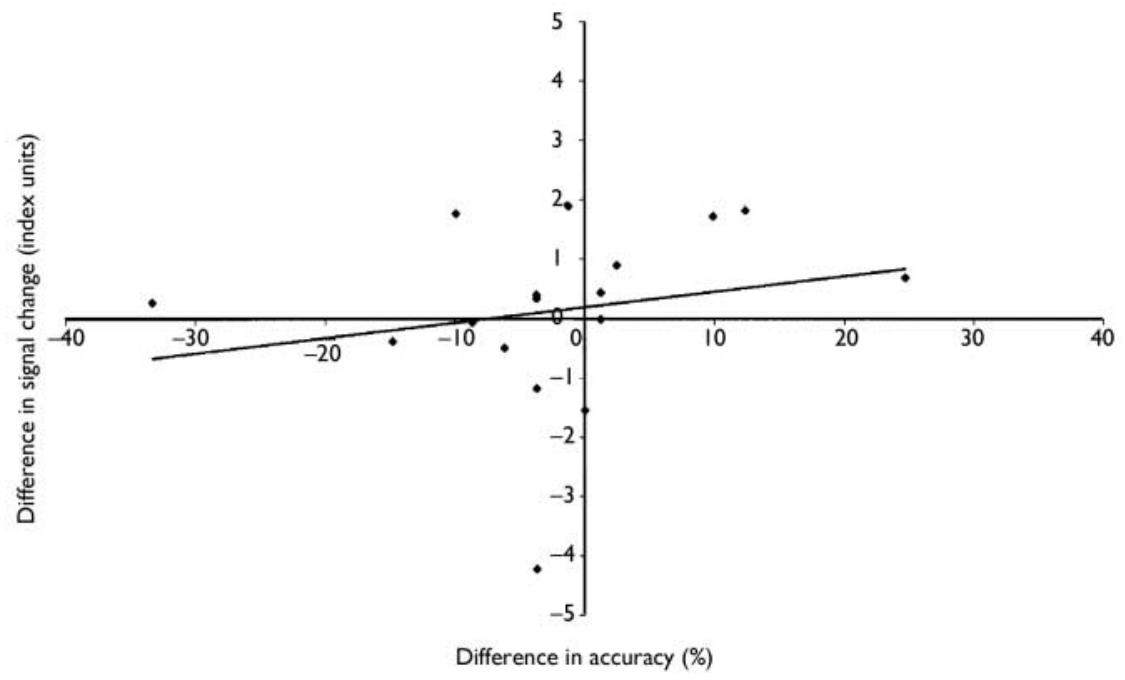

Fig. 2 Modafinil v. placebo in a working memory task: correlation between difference in anterior cingulate activation and behavioural performance. For each patient $(n=17)$ the $x$-axis shows difference in accuracy during an intra-scanner working memory task between modafinil and placebo conditions; the $y$-axis shows the corresponding difference in estimated functional magnetic resonance imaging (fMRI) signal change. Positive values indicate greater accuracy/greater fMRI signal change on modafinil than on placebo.

when these patients were excluded from the group analysis, the latter was no longer significant (for 12 patients receiving 'atypicals', $\rho=0.03$; one-tailed $P=0.47$ ). Exclusion of those subjects whose performance was at the level of chance on placebo led to exclusion of three of the four subjects receiving depots and a reduction of the anterior cingulate cortex/performance correlation to trend significance $(\rho=0.58$; one-tailed $P=0.06$ ). Hence, it is possible that those receiving depot medication derived the greatest benefit from modafinil (perhaps as a consequence of greater initial deficit) and contributed most to the findings.

We further investigated this post hoc 'deficit/benefit' hypothesis and found that those exhibiting improved working memory performance on modafinil had demonstrated significantly worse performance on a verbal fluency task at initial assessment compared with other patients. Responders generated 7-18 words in 1 min (median $10)$; non-responders generated 4-25 words, median 11.5 (Mann-Whitney $U$-test; $Z=2.19 ; P=0.03)$. There were no correlations between intra-scanner working memory task performance and specific symptom ratings.

Finally, by way of validating our sample against those described in successive reports, which have repeatedly described positive correlations between 2-back accuracy and right dorsolateral prefrontal cortex activity in people with schizophrenia compared with 'normals' (Callicott et al,
1999, 2000), we examined the correlates of performance per se on the 2-back task. We found the right dorsolateral prefrontal cortex implicated under both conditions (placebo; $x=44 \mathrm{~mm}, y=41 \mathrm{~mm}, z=9 \mathrm{~mm}$, 21 voxels, $Z=2.75, P<0.01$, uncorrected, for height of activation; modafinil: $x=44 \mathrm{~mm}, y=49 \mathrm{~mm}, z=18 \mathrm{~mm}, 62$ voxels, $Z=3.15, P<0.01$, uncorrected, for height of activation)

\section{DISCUSSION}

\section{Neural response}

We administered a single low dose of modafinil to people with chronic schizophrenia and assessed working memory during fMRI. Our data reveal a specific effect of the drug upon an area of the frontal lobe called the anterior cingulate cortex. Importantly, this effect was specific to the active memory condition and not the baseline state. Anterior cingulate cortex is involved in a wide range of executive functions (Bush et al, 2000) and has been activated during working memory protocols in previous studies (Callicott et al, 2000, 2003; Meyer-Lindenberg et al, 2001). Its activation in people with chronic schizophrenia appears to be enhanced by modafinil (Fig. 1).

\section{Cognitive response}

Despite enhanced anterior cingulate cortex activation at the group level (during working memory performance), most of our patients did not exhibit enhanced cognition. For the group as a whole there was no effect of modafinil upon working memory (on this protocol). Therefore it might be posited that increased anterior cingulate cortex activation is either unrelated to cognition or indicative of reduced efficiency of cognitive processing (Callicott et al, 2000; Manoach, 2003). If greater anterior cingulate cortex activation occurs without an increase in cognitive performance then brain function might be said to be less efficient in the presence of modafinil. However, it is important to note that cognitive function did improve in a minority of patients, and that although it might be posited that this is to be expected statistically (as merely a manifestation of variation around the mean), there is a feature of the data that runs counter to this interpretation. This is the positive correlation between anterior cingulate cortex activation and cognitive improvement (Fig. 2). If modafinil simply made anterior cingulate cortex function less efficient, then we should expect such a correlation to be negative. The positive correlation implies that the magnitude of anterior cingulate cortex activation and cognitive performance are indeed related in the context of modafinil exposure; those exhibiting the greater anterior cingulate cortex response also exhibit the greater cognitive enhancement (of working memory).

\section{Accounting for 'responders'}

It is of interest that those exhibiting greater physiological and cognitive response to modafinil tended to be those who were receiving 'typical' neuroleptic medications (depots and sulpiride). The numbers are small but there may be a rationale for this finding. The neurotransmitter systems implicated in the promotion of wakefulness by modafinil include the dopaminergic and serotonergic systems. Although antipsychotics share antagonism of the dopaminergic system (especially at the $\mathrm{D} 2$ receptor), the typicals and atypicals differ in their affinities for other receptors, particularly $5 \mathrm{HT}_{2 \mathrm{~A}}$ (Keefe et al, 1999). In animal models modafinil's effect of increasing alertness is attenuated by $5 \mathrm{HT}_{2 \mathrm{~A}}$ antagonism (Shelton et al, 1995), hence, it is possible that atypical antipsychotic treatment (if antagonising $5 \mathrm{HT}_{2 \mathrm{~A}}$ ) might constrain the cognotropic effects of modafinil (a hypothesis deserving further study). Alternatively, the ability of atypicals 
themselves to enhance cognition (albeit to a limited extent; Keefe et al, 1999) might curtail further improvement (on modafinil).

\section{Safety}

There are a number of caveats to our study and its findings. First, for the reasons described, we deliberately utilised a relatively low dose of modafinil. This may have been sub-optimal. Nevertheless, one of our participants underwent psychotic relapse 4 days post-drug. We cannot determine whether modafinil was responsible. Although covert non-adherence to concurrent medication cannot be excluded (he was receiving an oral antipsychotic), we must be wary of seeking to exculpate the test medication. However, a psychotic reaction to a single dose of modafinil is unprecedented (to our knowledge), although there are cases of psychosis emerging de novo in those receiving multiple doses. The manufacturer's prescribing information reports that: 'one healthy male volunteer developed ideas of reference, paranoid delusions, and auditory hallucinations in association with multiple daily $600 \mathrm{mg}$ doses of [modafinil] and sleep deprivation. There was no evidence of psychosis 36 hours after drug discontinuation.' (Cephalon, 1999). The British National Formulary does not mention psychosis as either a contraindication to or a consequence of modafinil exposure.

In a recent study of 20 patients with schizophrenia exposed to a single dose of $200 \mathrm{mg}$ of modafinil, the drug elicited no exacerbation of psychosis (Turner et al, 2004). However, there are anecdotal accounts of schizophrenic relapse following repeated dosing (Narendran et al, 2002; Rosenthal \& Bryant, 2003). Further multi-dosing studies in other centres may help to clarify the magnitude of such a putative risk.

\section{ACKNOWLEDGEMENTS}

We thank the patients who participated in this study, Dr C. W. Lawson, radiography colleagues at the Royal Hallamshire Hospital, Ms Anne Connors (pharmacist), the nursing staff on Rowan Ward (Clinical Academic Unit) and Ms Karen Roy for initial advice. The sponsor was involved in scientific discussions regarding study design but had no role in data collection, data analysis, data interpretation, the writing of the report or the decision to submit the paper for publication. We are grateful to the University of Sheffield and Sheffield Care Trust for insurance and indemnification of this study.

\section{CLINICAL IMPLICATIONS}

- Schizophrenia is associated with cognitive impairments that have an impact upon social function; a cognitive enhancer such as modafinil might reduce such impairments.

Patients with chronic schizophrenia exhibited increased anterior cingulate activation in response to modafinil while performing a working memory protocol; some also exhibited improved memory performance.

Of the patients exhibiting improved memory performance on modafinil, most were receiving concomitant 'typical' antipsychotic medication.

\section{LIMITATIONS}

- As in many neuroimaging studies, the number of subjects examined is relatively small.

- The impact of memory improvement upon day-to-day function (outside the scanner) remains to be demonstrated.

- Those patients who may derive cognitive benefit from modafinil require further characterisation.

SEAN A. SPENCE, MRCPsych, RUSSELL D. GREEN, MBChB, Sheffield Cognition and Neuroimaging Laboratory (SCANLab), Academic Clinical Psychiatry, Division of Genomic Medicine, University of Sheffield; IAIN D. WILKINSON, PhD, Academic Unit of Radiology, University of Sheffield; MIKE D. HUNTER, MRCPsych, SCANLab, Academic Clinical Psychiatry, Division of Genomic Medicine, University of Sheffield, Sheffield, UK

Correspondence: Dr Sean A. Spence, Sheffield Cognition and Neuroimaging (SCANLab), Academic Clinical Psychiatry, Division of Genomic Medicine, University of Sheffield, The Longley Centre, Norwood Grange Drive, Sheffield S5 7JT, UK. Tel: +44 (0) II4 22 61519; Fax: +44 (0) II4 2261522 ; E-mail: s.a.spence@sheffield.ac.uk

(First received 18 May 2004, final revision 19 October 2004, accepted 21 October 2004)

\section{REFERENCES}

American Psychiatric Association (1994) Diagnostic and Statistical Manual of Mental Disorders (4th edn) (DSM-IV). Washington, DC: APA.

Andreasen, N. C. (1983) Scale for the Assessment of Negative Symptoms (SANS). lowa City: University of lowa.

Andreasen, N. C. (1984) Scale for the Assessment of Positive Symptoms (SAPS). lowa City: University of lowa.

Barnes, T. R. E. (1989) A rating scale for drug-induced akathisia. British Journal of Psychiatry, 154, 672-676.

Beck, A. T., Ward, C. H., Mendelson, M., et al (1961) An inventory for measuring depression. Archives of General Psychiatry, 4, 561-57I.

Bertolino, A., Sciota, D., Brudaglio, F., et al (2003) Working memory deficits and levels of $N$ acetylaspartate in patients with schizophreniform disorder. American Journal of Psychiatry, 160, 483-489.

British Medical Association (2004) British National Formulary. London: British Medical Association/Royal Pharmaceutical Society of Great Britain.
Bush, G., Luu, P. \& Posner, M. I. (2000) Cognitive and emotional influences in anterior cingulate cortex. Trends in Cognitive Sciences, 4, 215-222.

Callicott, J. H., Ramsey, N. F., Tallent, K., et al (1998) Functional magnetic resonance brain mapping in psychiatry: methodological issues illustrated in a study of working memory in schizophrenia. Neuropsychopharmacology, 18, 186-196.

Callicott, J. H., Mattay, V. S., Bertolino, A., et al (1999) Physiological characteristics of capacity constraints in working memory as revealed by functional MRI. Cerebral Cortex, 9, 20-26.

Callicott, J. H., Bertolino, A., Mattay, V. S., et al (2000) Physiological dysfunction of the dorsolateral prefrontal cortex in schizophrenia revisited. Cerebral Cortex, 10, 1078-1092

Callicott, J. H., Egan, M. F., Mattay, V. S., et al (2003) Abnormal fMRI response of the dorsolateral prefrontal cortex in cognitively intact siblings of patients with schizophrenia. American Journal of Psychiatry, 160 709-719.

Cephalon (1999) Prescribing Information: Provigil (Modafinil) Tablets. West Chester, PA: Cephalon, Inc. 
Evans, A. C., Collins, D. L., Mills, S. R., et al (1993) 3D statistical neuroanatomical models from $305 \mathrm{MRI}$ volumes. IEEE Nuclear Science Symposium Medical Imaging Conference Proceedings, 108, 1877-1878.

Folstein, M. F., Folstein, S. E. \& McHugh, H. R. (1975) 'Mini-Mental State': a practical method for grading the cognitive state of patients for the clinician. Journal of Psychiatric Research, 12, 189-198.

Friston, K. J., Ashburner, J., Frith, C. D., et al (1995) Spatial registration and normalisation of images. Human Brain Mapping, 2, 165-169.

Friston, K. J., Holmes, A. P. \& Worsley, K. J. (1999) How many subjects constitute a study? Neurolmage, $\mathbf{1 0}$ $1-5$.

Guy, W. (1976) Early Clinical Drug Evaluation Unit Assessment Manual for Psychopharmacology, Revised Edition. Washington, DC: Department of Health, Education, and Welfare.

Keefe, R. S. E., Silva, S. G., Perkins, D. O., et al (1999) The effects of atypical antipsychotic drugs on neurocognitive impairment in schizophrenia: a review and meta-analysis. Schizophrenia Bulletin, 25, 201-222.

Lin, J.-S., Hou, Y. \& Jouvet, M. (1996) Potential brain neuronal targets for amphetamine-, methylphenidateand modafinil-induced wakefulness, evidenced by c-fos immunocytochemistry in the cat. Proceedings of the National Academy of Sciences USA, 93, 14128-14133.
Logothetis, N. K., Pauls, J., Augath, M., et al (200I) Neurophysiological investigation of the basis of the fMR signal. Nature, 412, 150-157.

Manoach, D. S. (2003) Prefrontal cortex dysfunction during working memory performance in schizophrenia: reconciling discrepant findings. Schizophrenia Research, 60, 285-298.

Manoach, D. S., Press, D. Z., Thangaraj, V., et al (1999) Schizophrenic subjects activate dorsolatera prefrontal cortex during a working memory task, as measured by fMRI. Biological Psychiatry, 45, II28-II37.

Meyer-Lindenberg, A., Poline, J-B., Kohn, P. D., et a (200I) Evidence for abnormal cortical functional connectivity during working memory in schizophrenia. American Journal of Psychiatry, 158, 1809-1817.

Narendran, R., Young, C. M., Valenti, A. M., et al (2002) Is psychosis exacerbated by modafinil? Archives of General Psychiatry, 59, 292-293.

Nelson, H. E. \& O'Connell, A. (1978) Dementia: the estimation of premorbid intelligence levels using the National Adult Reading Test. Cortex, 14, 234-244.

Overall, J. \& Gorham, D. (1962) The Brief Psychiatric Rating Scale. Psychological Reports, 10, 799-812.

Robertson, P. \& Hellriegel, E.T. (2003) Clinical pharmacokinetic profile of modafinil. Clinical Pharmacokinetics, 42, 123-137.
Rosenthal, M. H. \& Bryant, S. (2003) Benefits of adjunct modafinil in an open-label, pilot study in patients with schizophrenia. Schizophrenia Research, 60, 301.

Scammell, T. E., Estabrooke, I.V., McCarthy, M.T., et al (2000) Hypothalamic arousal regions are activated during modafinil-induced wakefulness. Journal of Neuroscience, 20, 8620-8628.

Shelton, J., Nishino, S., Vaught, J., et al (1995) Comparative effects of modafinil and amphetamine on daytime sleepiness and cataplexy of narcoleptic dogs. Sleep, 18, 817-826.

Simpson, G. N. \& Angus, J.W. S. (1970) A rating scale for extrapyramidal side effects. Acta Psychiatrica Scandinavica, 212 (suppl. 44), II-19.

Talairach, P. \& Tournoux, J. A. (1988) A Stereotactic Co-planar Atlas of the Human Brain. Stuttgart: Thieme.

Turner, D. C., Clark, L., Pomarol-Clotet, E., et al (2004) Modafinil improves cognition and attentional set shifting in people with schizophrenia. Neuropsychopharmacology, 29, 1363-1373.

US Modafinil in Narcolepsy Multicenter Study Group (2000) Randomised trial of modafinil as a treatment for the excessive daytime somnolence of narcolepsy. Neurology, 54, II66-1175.

Velligan, D. I., Mahurin, R. K., Diamond, P. L., et al (1997) The functional significance of symptomatology and cognitive function in schizophrenia. Schizophrenia Research, 25, 2I-3!. 